\title{
O PROCESSO DE REABILITAÇÃO DE PESSOAS PORTADORAS DE LESÃO MEDULAR BASEADO NASTEORIAS DE ENFERMAGEM DE WANDA HORTA, DOROTHEA OREM E CALLISTA ROY: UM ESTUDO TEÓRICO
}

[The rehabilitation process of people suffering from spinal cord injury based on the nursing theories of Wanda Horta, D orothéa O rem and Callista Roy: a theoretical study]

[El proceso de la rehabilitación de las personas con lesión del cordón espinal (medular) basado en las teorías de enfermería de Wanda Horta, Dorothéa O rem y Callista Roy: un estudio teórico]

\author{
Janaina Vall* \\ Kátia Isabel Lima L emos** \\ A ndréa Socorro Idalino J anebro***
}

\begin{abstract}
RESUM O: Estudo teórico que correl aciona as Teorias das Necessidades Humanas Básicas de Wanda Horta, Teoria do A utocuidado de Dorothea Orem e Teoria da A daptação de Callista R oy com o processo de reabilitação de pessoas portadoras de lesão medular. A definição de um marco conceitual é um processo complexo mas também essencial para a qualidade da assistência de enfermagem prestada a esses pacientes, que enfrentam a necessidade de recuperação de sua independência, reinserção social e restabelecimento da saúde mental. Por isso a decisão de fundamentar sua prática assistencial nas teorias de enfermagem é de grande importância para os enfermeiros reabilitadores.
\end{abstract}

PALAVRAS-CHAVE: Enfermagem em reabilitação; Teoria de enfermagem; M odelos de enfermagem.

\section{INTRODUÇÃO}

A lesão medular é uma grave síndrome neurológica incapacitante que se caracteriza por alterações da motricidade, sensibilidade superficial e profunda e distúrbios neurovegetativos dos segmentos do corpo local izados abaixo da lesão. A grande maioria das pessoas acometidas por tal lesão, agora em cadeira de rodas, necessita passar por um processo de reabilitação que 0 ajude a atingir seu melhor potencial físico, psicológico e social, vocacional e educacional, compatível com seu déficit fisiológico,

\footnotetext{
*Enfermeira, M estre em Enfermagem pela Universidade Federal do Ceará.

**Enfermeira, M estranda do Programa de Pós-graduação em Vigilância Sanitária da Universidade Estadual do Ceará.

***Enfermeira, M estranda do Programa de Pós-graduação em

Vigilância Sanitária da Universidade Estadual do Ceará.
}

anatômico, limitações ambientais, desejos e planos de vida. R eabilitação é um conceito que deve envolver todo o sistema de saúde e integrar ou reintegrar na sociedade ativamente a pessoa cuja capacidade esteja diminuída ${ }^{(1)}$. Dentre os objetivos desejados através da reabilitação estão: aumento da independência, diminuição do tempo de internação e, principalmente, mel hora na qualidade de vida.

Observa-se que 0 enfermeiro tem papel fundamental neste processo, como membro da equipe interdisciplinar, sempre considerando o paciente como um ser individual com uma história de vida própria, com características próprias, que podem determinar de forma decisiva as capacidades funcionais e psicossociais preservadas para serem trabal hadas na sua reabilitação. A través da reabilitação, pessoas com incapacidades físicas são capacitadas para mobilizar recursos, decidir o que elas desejam, o que são capazes de ser e al cançar metas mediante seus próprios esforços, em seus próprios caminhos ${ }^{(2)}$.

No entanto, para que a enfermagem atue eficientemente neste processo, necessita desenvolver sua metodologia de trabal ho fundamentada no método científiCo ${ }^{(3)}$. Há um esforço, nos dias presentes, no sentido de empregar uma metodologia científica que leva a uma assistência individual izada, planejada, qualificada e científica ${ }^{(4)}$. A investigação científica deve ser guiada pela teoria ${ }^{(5)}$ e pelo conhecimento, entendido como um processo ${ }^{(6)}$. A s teorias de enfermagem são importantes para o desenvolvimento da profissão como ciência (7) e porque são um guia para novas buscas, por mais claras e apuradas visões do mundo, substituindo as visões fornecidas pel o senso comum ${ }^{(8)}$. Por isso é importante que num Centro de Reabilitação, os enfermei ros trabal hem, buscando apoio nas teorias previamente elaboradas por estudiosos que marcaram a história da enfermagem, a fim de embasar o cuidado que prestam no processo de reabilitação.

A s teorias cumprem a função de descrever, explicar

Cogitare Enferm 2005 set/dez; 10(3):63-70 
e fazer previsões sobre fenômenos específicos. Já o modelo conceitual é análogo ao projeto arquitetônico de uma casa. É um grupo de conceitos inter-relacionados que se encaixam em virtude de sua relevância para um tema ou molde comum ${ }^{(9)}$. Os marcos conceituais oferecem subsídios para a prática profissional ${ }^{(10)}$ e a aplicabilidade de uma teoria de enfermagem permite explicitar os propósitos, contextos, variáveis, explicações teóricas, evidência empírica e a utilização de novas abordagens na prática de enfermagem que determinam a natureza dos seus el ementos descritivos ${ }^{(11)}$. Os significados das teorias para a profissão de enfermagem suscitam uma postura al erta e comprometida com a intenção científica que se volta para uma autocompreensão, retroalimentação e anál ise de pressupostos e das bases teórico-filosóficas de afirmação da enfermagem como corpo de conhecimento posto a serviço da formação, exercício e atualização profissional e em benefício da humanidade ${ }^{(12)}$. N este contexto, optamos trabal har 0 marco conceitual neste estudo de reflexão.

$H$ á necessidade de novos conceitos compatíveis com a cultura brasileira no que se refere ao ambiente, ao homem, ao processo saúde-doença, à enfermagem eao cuidado ${ }^{(13)}$. Quando se fala em assumir um marco conceitual, 0 enfermeiro está buscando uma cientificação profissional e a melhora na qualidade da assistência prestada ao ser humano ${ }^{(14)}$, por isso é importante escolher um marco para utilização na prática assistencial ${ }^{(15)}$. A utilização deste é importante também como um meio para esclarecer propósitos, orientar funções e assegurar resultados ${ }^{(16)}$. A definição de um marco e de uma metodologia de enfermagem para a prática é um processo tridimensional que envolve 0 pensar, o fazer e o sentir, tornando a sua implementação para a prática assistencial, um processo complexo e contínuo ${ }^{(17)}$

M uitas vezes, teoria e prática percorrem trajetórias conflitantes, no entanto, necessitam estar inter-relacionadas, pois toda prática está atrelada à teoria, e esta sem a prática, se torna estéril (18).

N este contexto, o cuidado de enfermagem baseado em modelos assistenciais é fundamental, pois, além de nortear a dinamização de recursos humanos e materiais, facilita a avaliação da assistência prestada. Dessa forma, é possível verificar o alcance de padrões mínimos de assistência, oferecendo subsídios aos indicadores de custos e rendimentos, indicando também áreas que requei ram aprimoramento. Este processo pode ser alcançado através do estudo das teorias já existentes, para que seja traçado um modelo próprio, peculiar de cada Instituição ${ }^{(19)}$. M uitas vezes, uma única teoria é levada à prática, porém, dependendo da característica da população atendida, pode ser necessário que se utilize, numa mesma instituição, mais de uma teoria, ou ainda que se crie uma nova.

Diante disso, para efetivar a reflexão estudamos as teorias de enfermagem como um todo e buscamos correlacionar seus pressupostos com o processo de reabili- tação de pessoas portadoras de lesão medular. L evamos em consideração o objetivo deste estudo, de demonstrar como e quais teorias de enfermagem se aplicam ao processo de reabilitação do portadores de lesão medular, a fim de alcançar um marco conceitual de assistência voltado para a reabilitação.

Para isso desenvolvemos uma revisão bibliográfica dos trabalhos existentes sobre o tema. As fontes de busca foram as bases de dados Lilacs e Medline, utilizando os termos "teoria de enfermagem" e "modelos de enfermagem". No entanto, devido às poucas referências encontradas quando real izado o cruzamento com o termo chave "enfermagem em reabilitação", os livros sobre as teorias existentes contribuíram substancialmente para nosso estudo.

Primeiramente, estudamos os princípios de enfermagem estipulados por Florence $\mathrm{N}$ ightingale, visto que esse foi o marco inicial da prática de enfermagem como profissão. A pós seguimos com o estudo das demais teorias, finalizando com a discussão de quais se aplicariam ao processo de reabilitação da pessoa portadora de lesão medular. A s teoristas estudadas foram: Hildegard Peplau, Virginia Henderson, Lydia Hall, D orothea O rem, D orothy J ohnson, F aye A bdellah, I da O rlando, M yra L evine, I mogene K ing, Callista R oy, J ean Watson, $M$ adeleine L eininger e $M$ argaret Newman ${ }^{(19,20)}$.

\section{CONSTRUINDO UM MARCO CONCEITUAL}

Os princípios da reabilitação para este estudo priorizam o ser com lesão medular (ser humano) como sujeito de ação e não como objeto (passivo), desenvolvem a assistência ao indivíduo, ficando a prevenção no repasse de informação e favorecendo a consciência de atuar dentro de seus direitos de ir e vir, desenvolvendo atitudes críticas e comportamentos que valorizem a qualidade de vida (assistência de enfermagem), promovendo a saúde e transformando o indivíduo em agente de mudança, em seu contexto de vida (ambiente).

Todas as teorias estudadas, de alguma maneira abordam estes princípios essenciais para o processo de reabilitação. N o entanto, al guns fatores limitantes fazem com que não se apliquem ao processo de reabilitação, conforme descritos no quadro 1. Vale ressaltar que as informações contidas nesse quadro contaram com nossa interpretação e experiência na área de reabilitação, visto que as considerações sobre as teorias podem variar conforme o referencial teórico adotado bem como não se aplicar a outros contextos, sujeitos ou pesquisadores.

Quadro 1 - Pressupostos das teorias estudadas e sua limitação para a prática reabilitadora, Ceará. 


\begin{tabular}{|c|c|c|}
\hline TEÓRICO & PRESSUPOSTOS & LIMITAÇÃO \\
\hline Hildegard Peplau & $\begin{array}{l}\text { O núcleo desta teoria é o processo interpessoal que } \\
\text { consiste nas fases de orientação, identificação, exploração e } \\
\text { resolução. Este processo é influenciado pelas percepções } \\
\text { individuais do paciente e do enfermeiro. }\end{array}$ & $\begin{array}{l}\text { Em um processo de reabilitação, não se pode } \\
\text { trabalhar com percepções individuais, mas } \\
\text { interdisciplinares e familiares. }\end{array}$ \\
\hline Lydia Hall & $\begin{array}{l}\text { A teoria de Hall envolve três círculos entrelaçados que } \\
\text { representam o cuidado direto ao paciente, a essência que } \\
\text { lida com os sentimentos e motivações do paciente e o } \\
\text { processo de cura. O principal instrumento utilizado pelo } \\
\text { enfermeiro é a reflexão. }\end{array}$ & $\begin{array}{l}\text { Trabalha com o processo de cura, muitas vezes } \\
\text { inatingível no processo de reabilitação. }\end{array}$ \\
\hline Faye Abdellah & $\begin{array}{l}\text { Utiliza a abordagem de solução de problemas com } \\
\text { problemas-chave relacionados com as necessidades de } \\
\text { saúde das pessoas, de acordo com a hierarquia de } \\
\text { necessidades de Maslow. São exploradas as formas de } \\
\text { usar os problemas no processo de enfermagem. }\end{array}$ & $\begin{array}{l}\text { Orientação centralizada na enfermagem e não } \\
\text { na equipe interdisciplinar de saúde. }\end{array}$ \\
\hline Myra Levine & $\begin{array}{l}\text { Sua teoria inter-relaciona os conceitos de adaptação, } \\
\text { conservação e integridade, numa visão da totalidade do } \\
\text { indivíduo. }\end{array}$ & $\begin{array}{l}\text { Trabalha com uma visão muito abrangente e } \\
\text { geral do contexto saúde-doença. Na } \\
\text { reabilitação não se trata doenças, mas se } \\
\text { reestabelece a independência e autonomia do } \\
\text { indivíduo, conforme seu potencial. }\end{array}$ \\
\hline Madeleine Leininger & $\begin{array}{l}\text { Teoria da diversidade e universalidade do cuidado cultural, } \\
\text { abordando a enfermagem transcultural. Apresenta a idéia de } \\
\text { que os padrões e os processos de cuidado possam ser } \\
\text { universais ou diversificados, tendendo, no entanto, à } \\
\text { diversificação. }\end{array}$ & $\begin{array}{l}\text { O processo de reabilitação vai muito mais além } \\
\text { da questão cultural, princípio único desta teoria. }\end{array}$ \\
\hline Virginia Henderson & $\begin{array}{l}\text { Sua teoria define a enfermagem como uma função } \\
\text { independente: a de agir para o paciente quando ele carece } \\
\text { de conhecimento, força física ou vontade de agir por si } \\
\text { mesmo. }\end{array}$ & $\begin{array}{l}\text { A função de enfermagem como independente } \\
\text { não condiz com a prática da reabilitação que } \\
\text { necessita de uma abordagem interdisciplinar. }\end{array}$ \\
\hline Dorothy Johnson & $\begin{array}{l}\text { Aborda o modelo de sistema comportamental, que é um } \\
\text { modelo de cuidados de enfermagem que defende o } \\
\text { incentivo do funcionamento comportamental eficiente e } \\
\text { efetivo do paciente para a prevenção da doença. }\end{array}$ & $\begin{array}{l}\text { Seu enfoque principal é na prevenção de } \\
\text { doenças. Na reabilitação trabalhamos com a } \\
\text { doença já instalada ou suas seqüelas. }\end{array}$ \\
\hline Ida Orlando & $\begin{array}{l}\text { Está enraizada na interação entre enfermeira e paciente num } \\
\text { local e ocasião específica. Diante desta situação, a } \\
\text { enfermeira decide a conduta a ser tomada. }\end{array}$ & $\begin{array}{l}\text { Na reabilitação, o paciente é sujeito e não } \\
\text { objeto da ação, então também tem poder de } \\
\text { decisão. }\end{array}$ \\
\hline Imogene King & $\begin{array}{l}\text { Teoria da obtenção de metas através da estrutura de três } \\
\text { sistemas abertos: pessoal, interpessoal e social, em troca } \\
\text { contínua com seus ambientes. Os principais conceitos dessa } \\
\text { teoria são a interação, a percepção, a comunicação, a } \\
\text { transação, o papel, o estresse e o crescimento e } \\
\text { desenvolvimento. }\end{array}$ & $\begin{array}{l}\text { O estabelecimento de metas mútuas pelo } \\
\text { paciente e equipe de saúde é muito importante, } \\
\text { principalmente para o bom desempenho nas } \\
\text { atividades de vida diária e diminuição do } \\
\text { estresse e ansiedade. No entanto, em muitos } \\
\text { casos, podem constranger os pacientes que, } \\
\text { limitados e com pouco potencial não } \\
\text { conseguem atingir essas metas. }\end{array}$ \\
\hline Jean Watson & $\begin{array}{l}\text { Vincula várias teorias úteis para a prática de enfermagem. A } \\
\text { enfermeira torna-se uma ativa co-participante na luta do } \\
\text { paciente pela auto-atualização. O paciente é colocado no } \\
\text { contexto da familia e da comunidade. Prioriza o cuidado } \\
\text { sem o uso de tecnologias. }\end{array}$ & $\begin{array}{l}\text { É limitante pelo fato de que, na reabilitação as } \\
\text { tecnologias são muito utilizadas, por serem } \\
\text { importantes ferramentas de ajuda. }\end{array}$ \\
\hline Margaret Newman & $\begin{array}{l}\text { Newman desenvolveu uma teoria de saúde como expansão } \\
\text { da consciência na qual a doença e a não-doença são } \\
\text { sintetizadas para formar uma nova visão de saúde. A saúde } \\
\text { é vista como a explicação do padrão subjacente da } \\
\text { pessoa-ambiente. }\end{array}$ & $\begin{array}{l}\text { Muitas vezes a doença não explica um padrão } \\
\text { de comportamento e pode instalar-se por } \\
\text { motivos genéticos ou traumáticos. }\end{array}$ \\
\hline
\end{tabular}


3TEORIASE SUAADEQUAÇÃO AO PROCESSO DE REABILITAÇÃO

A pós minucioso estudo e discussão, optamos por três teorias para embasar o processo de reabilitação, construindo um marco conceitual de assistência de enfermagem. 0 critério utilizado para a escolha dos modelos foi a proximidade da teoria com a experiência vivenciada por nós em reabilitação. Desta maneira as teorias selecionadas foram: de Wanda Horta, Callista R oy e Dorothea Orem.

\subsection{TEORIA DAS NECESSIDADES HUMANAS BÁSI- CAS (WANDA HORTA)}

A teoria de Wanda Horta foi desenvolvida através da teoria da motivação humana, de M aslow, que se fundamenta nas necessi dades humanas básicas. Está embasada e engl oba leis que regem os fenômenos universais, tais como:

- L ei do equilíbrio (homeostase ou homeodinâmica): todo universo se mantém por processos de equilíbrio dinâmico entre os seus seres;

- Lei da adaptação: todos os seres do universo interagem com o seu meio externo, buscando sempre formas de ajuste para se manterem em equilíbrio;

- Lei do holismo: o universo é um todo, o ser humano é um todo, a célula é um todo, esse todo não é mera soma das partes constituintes de cada ser ${ }^{(22)}$.

Wanda H orta descreve também as necessi dades humanas básicas do ser humano, essenciais a sua sobrevivência no mundo. A s necessidades humanas básicas são estados de tensões, conscientes ou inconscientes, resultantes dos desequilíbrios hemodinâmicos dos fenômenos vitais. Em estados de equilíbrio dinâmico, as necessidades não se manifestam, porém estão latentes e surgem com maior ou menor intensidade, dependendo do desequilíbrio instalado. São aquelas condições ou situações que o indivíduo, família e comunidade apresentam decorrentes do desequilíbrio de suas necessidades básicas que exijam uma resolução, podendo ser aparentes, conscientes, verbalizadas ou não. São exemplos de necessidades psicobiológicas: oxigenação, nutrição, sono e repouso, abrigo, integridade física, sexualidade, locomoção e eliminação. São exemplos de necessidades psicosociais: segurança, amor, liberdade, lazer, auto-estima, comunicação, independência, entre outras ${ }^{(7,14,23)}$.

Em relação à aplicação desta teoria na prática reabilitadora, optamos por selecioná-la visto que se adequa principalmente à fase aguda das lesões medulares, na qual os indivíduos são muito dependentes de terceiros e quando chegam para o programa de reabilitação também são dependentes dos profissionais de saúde.

Neste momento, praticamente todas as necessidades humanas básicas são alteradas, visto que os pacientes apresentam distúrbios da oxigenação, nutrição, sono e re- pouso, integridade física (principalmente cutânea), sexualidade, locomoção e eliminação vesical e intestinal. N esta fase, é de responsabilidade também do enfermeiro suprir estes desequilíbrios, promovendo o esvaziamento vesical e intestinal, mediante cateterismos ou estímulos, realizar mudança de decúbito no leito, higiene corporal e outras atividades. No entanto, já nesta fase o enfermeiro deve enxergar o potencial deste paciente para 0 autocuidado.

A lém disso, outras necessidades psicosociais também se alteram como a segurança, liberdade, lazer, autoestima, independência, entre outras, as quais devem ser supridas com a ajuda do enfermeiro, quando a pessoa é incapaz de promover o autocuidado e devem ser fornecidas em quantidade e qualidade necessárias.

Todas as necessidades estão intimamente inter-relacionadas, uma vez que fazem parte de um todo: o ser humano. É claro que se pode perceber a inter-relação mais estreita entre al gumas necessidades e 0 distanciamento de outras, mas em maior ou menor intensidade todas elas sofrem alterações quando qual quer uma se manifesta, seja por desequilíbrio causado por falta ou excesso de demandas. Sendo assim, a teoria das N ecessidades Humanas Básicas se aplica ao processo de reabilitação visto que as necessidades psicobiológicas e sociais estão afetadas após uma lesão medular, daí a importância do enfermeiro na reconstrução do equilíbrio orgânico e mental, auxiliando e incentivando 0 autocuidado, conforme potencial do paciente para tal.

\subsection{A TEORIA DA ADAPTAÇÃO (CALLISTA ROY)}

De acordo com Callista Roy, o homem é um ser biopsicossocial em constante interação com as mudanças ambientais. No entanto, essas ações sistemáticas são desenvolvidas com o objetivo de promover as respostas adaptativas em situações de saúde e de doença. Essas respostas adaptativas afetam positivamente a saúde $(7,13,20)$.

Esta teoria tem como objetivo, promover a adaptação do homem em situações de saúde e doença, ou seja; promover a saúde da pessoa oportunizando as respostas adaptativas, o que vai ao encontro do propósito dos Centros de R eabilitação.

Os quatro el ementos essenciais do modelo de adaptação de R oy são:

A Pessoa - é o receptor do cuidado de enfermagem que, por sua vez, pode ser uma pessoa, uma família, uma comunidade ou uma sociedade. Cada um deles é considerado pela enfermeira um sistema adaptativo holístico, sendo o sistema e o ambiente baseado na troca de informações, matéria e energia.

Roy identificou quatro modos adaptativos ou categorias para a investigação de comportamentos que resultam dos mecanismos regulador e cognato. Esses modos são o fisiológico, o autoconceito, a função do papel e a interdependência. 
1. Modo fisiológico - representa a resposta física aos estímulos ambientais, e envolve, primariamente, 0 subsistema regulador. A necessidade básica desse modo fisiológico é a integridade fisiológica e é composta das necessidades associadas a oxigenação, a nutrição, a eliminação, a atividade e o repouso e a proteção. Os processos complexos deste modo estão associados com os sentidos, 0 fluido e os eletrólitos, a função neurológica e a função endócrina.

2. Modo de autoconceito - relaciona-se com a necessidade básica de integridade psíquica. Seu enfoque está associado a aspectos psicológico e espiritual da pessoa. 0 eu físico tem componentes de sensação e imagem corporal. 0 eu pessoal tem componentes próprios de autocoerência, de auto-ideal e de ser moral-ético-espiritual.

3. M odo de função do papel - identifica os padrões de interação social da pessoa em relação aos outros refletidos pel os papéis primário, secundário e terciário. A principal necessidade preenchida é a integridade social.

4. M odo de interdependência - é onde as necessidades afetivas são preenchidas. Este modo identifica os padrões de valor humano, afeição, amor e afirmação. Esses processos ocorrem através dos relacionamentos interpessoais tanto em nível individual quanto em grupo.

A mbiente - os estímulos do interior da pessoa e os estímulos em torno da pessoa representam o elemento do ambiente. 0 ambiente é definido como todas as condições, circunstâncias e influências que circundam e af etam o desenvolvimento e o comportamento de pessoas e grupos ${ }^{(20)}$.

Saúde - é definida como um estado e um processo de ser, de tornar-se uma pessoa total e integrada. A integridade da pessoa é expressa como a capacidade de preencher as metas de sobrevivência, crescimento, reprodução e domínio.

Dentro do modelo de adaptação de R oy, o conceito de saúde está intrinsecamente ligado ao de pessoa, de ambiente e de adaptação. Isso porque num enfrentamento, a pessoa necessita liberar energia para poder se adaptar, meIhorando dessa maneira, a saúde e promovendo a cura. É essa liberação de energia que une os conceitos de adaptação e saúde (20).

M eta de Enfermagem - é a promoção de respostas adaptativas em relação aos quatro modos adaptativos. Estas respostas são as que afetam positivamente a saúde. A enfermagem procura reduzir as respostas ineficientes e promove as respostas adaptativas como comportamento de saída da pessoa. 0 enfermeiro, portanto, promove a saúde em todos os processos da vida, incluindo o morrer com dignidade ${ }^{(20,22,26)}$.

A teoria de Callista R oy se aplica aos portadores de lesão medular visto que, após a fase aguda da doença, onde ele experiência sentimentos de raiva, cul pa e/ou barganha, tende a adaptar-se à situação, visto que a adaptação é uma característica inerente ao ser humano. N esta fase, a equipe interdisciplinar de reabilitação deve fornecer o suporte ade- quado, principalmente com apoio emocional e psicológico, tanto ao paciente quanto à sua família.

\subsection{TEORIA DO AUTO-CUIDADO (DOROTHEA OREM)}

A teoria de enfermagem de Orem proporciona uma base compreensiva para a nossa prática de Enfermagem que vai permear o desenvolvimento do indivíduo/família/comunidade nas ações de autocuidado. Dorothea Orem é uma teorista de enfermagem que está contribuindo, significativamente, com a construção de uma base conceitual que amplia o corpo de conhecimentos de enfermagem ${ }^{(7,8,13)}$. Nesse contexto, Orem desenvolveu a teoria de enfermagem do déficit do autocuidado que é composta de três teorias inter-relacionadas ${ }^{(24)}$ :

\subsubsection{Teoria do Autocuidado}

- A utocuidado: é o desempenho ou a prática de atividades da vida diária que o indivíduo realiza em seu benefício para manter a vida, a saúde e o bem-estar. Para tanto, o indivíduo tem de ser capaz de engajar-se no autocuidado, tomando como requisitos as atividades de vida diária (manutenção de ingesta hídrica, ar, alimentos, manutenção do equilíbrio entre a solidão e interação social, dentre outros).

- A ção do autocuidado: é a capacidade humana ou 0 poder de engajar-se no autocuidado para atingir as suas necessidades da vida diária. Essa capacidade é afetada por fatores:

1) condicionantes básicos: idade, sexo, estado de desenvolvimento, estado de saúde, orientação sócio-cultural, fatores ambientais, padrão de vida, entre outros.

2) demanda terapêutica - ação desempenhada por alguns membros de um grupo social para provocar eventos e resultados que beneficiem outros de maneira específica.

- Requisitos ou exigências do autocuidado: São comuns a todos os seres humanos durante todos os estágios do ciclo de vida e devem ser vistos como fatores inter-relacionados, cada um af etando os outros. Esses requisitos são as atividades da vida diária.

- 0 autocuidado no desvio de saúde é exigido em condições de doença ou de lesão ou pode resultar das medidas exigidas para diagnosticar ou corrigir um problema identificado.

\subsubsection{Teoria do Déficit de Autocuidado}

Ocorre quando há incapacidade ou limitação do paciente, cuidador ou família em manter 0 autocuidado efetivo e continuado e com isso, delineia a presença da enfermagem na assistência. O rem identifica cinco métodos de ajuda:

\footnotetext{
- A gir ou fazer para outra pessoa;

- Guiar e orientar;
} 
- Proporcionar apoio físico e psicológico;

- Proporcionar e manter em ambiente de apoio ao desenvolvimento pessoal;

- Ensinar.

Dessa maneira, o enfermeiro pode ajudar o indivíduo, usando um ou todos esses métodos para proporcionar uma assistência voltada ao autocuidado.

\subsubsection{Teoria dos Sistemas de E nfermagem}

Orem também discute em sua teoria, cada um dos quatro principais conceitos: ser humano, saúde, sociedade e enfermagem. Para delinear seu trabal ho, o enfermeiro leva o conjunto da sua assi stência em comum acordo com o paciente, uma vez que na fase de investigação, el e coleta dados baseados no estado de saúde da pessoa, perspectiva da pessoa sobre sua saúde e exigências de autocuidado da pessoa, dentre outros.

A teoria dos sistemas também está baseada nas necessidades de autocuidado e nas capacidades do paciente para desempenhar as atividades. Se existir dificuldade, limitação ou déficit para manter esse desempenho então a assistência da enfermagem deve estar presente ${ }^{(25)}$.

Orem identificou três classificações de sistemas de enfermagem para preencher os requisitos de autocuidado do paciente ${ }^{(26)}$ :

1. Sistema total mente compensatório - é representado pela situação em que o indivíduo é incapaz de "engajarse nas ações de autocuidado, seja pela limitação ou pela restrição às atividades da vida diária. São dependentes de terceiros para contribuir para o seu bem-estar e qualidade de vida.

2. Sistema parcialmente compensatório - é representado por tudo que o indivíduo consegue fazer, mas ainda precisa de auxílio de terceiros para atingir o seu bemestar.

3. Sistema de apoi o-educação - nesse sistema o indivíduo é capaz de desempenhar, ou pode e deve aprender a desempenhar as medidas exigidas pelo autocuidado.

Essas premissas vão ao encontro da teoria de Wanda Horta, em que "assistir o paciente" significa fazer pelo ser humano aquilo que ele não pode fazer por si mesmo, ajudar ou auxiliar, quando parcialmente impossibilitado de se autocuidar. A lém disso, orientar ou ensinar, supervisionar e encaminhar a outros profissionais, quando necessário. 0 enfermeiro, juntamente com outros profissionais, é responsável pela assistência ao ser humano no atendimento de suas necessidades básicas, visando torná-lo independente dessa assistência, valorizando o ser humano como um todo, quando possível, prestando cuidados preventivos, curativos e de reabilitação, tornando o paciente participante ativo no seu autocuidado. E sses cuidados devem ser realizados de forma sistemática, através da utilização do Processo de Enfermagem que deve ser adaptado às condições de cada local.
Em relação à aplicação desta teoria na prática de reabilitação, optamos por selecioná-la visto que se adequa principalmente ao objetivo principal da reabilitação que é 0 alcance da independência proporcionando melhora da qualidade de vida dos pacientes com limitações físicas.

0 paciente portador de lesão medular, depois de atingido o processo de adaptação, está pronto para adquirir maior independência em seu cotidiano. N esta fase, o enfermeiro e toda a equipe interdisciplinar de saúde já podem incentivá-lo para o autocuidado através, principalmente, das atividades educativas e treino deAV D 's (A tividades de Vida Diária). É preciso que a equipe de saúde priorize ações educativas, ministrando aulas sobre cuidados com a pele, reeducação vesical, intestinal e sexual, entre outras. A lém disso, é importante promover treinos de higiene, transferência, vestuário, marcha em terrenos regulares e acidentados, entre outras. Todas estas atividades levam o paciente a exercer 0 autocuidado. N os casos de pacientes incapacitados para o autocuidado, como é o caso dos tetraplégicos, estas mesmas orientações devem ser fornecidas e trabal hadas juntamente com os familiares e/ou cuidadores.

\section{CONSIDERAÇÕES FINAIS}

É de grande importância o esforço por parte da enfermagem em trabalhar, unindo teoria à prática porque, quando se cuida de seres humanos, devem ser observados não apenas os aspectos etiológicos, patológicos e clínicos, mas analisa-se também a total conseqüência das limitações do indivíduo na realidade psicossocial de sua família. A identificação destas incapacidades, que é a conseqüência da lesão funcional ou anatômica secundária ao trauma ou doença, que acarreta dificul dades ou mesmo impede 0 desempenho de uma determinada função, é de suma importância para a reabilitação. A partir do momento em que se conhecem estas limitações, pode-se planejar a assistência, traçando objetivos e metas a serem al cançados, que deverão ser dirigidas à independência nas capacidades preservadas, treino familiar se necessário e a prevenção de seqüelas, o que determina a efetivação do processo de reabilitação. Trabal har tendo estabelecido um marco conceitual, auxilia e orienta o percurso a ser feito, impõe parâmetros e nos deixa mais seguros ${ }^{(29)}$.

A eficácia e continuação do processo de reabilitação na vida cotidiana, dependem da soma de conhecimentos e decisões do paciente, sua família e equipe interdisciplinar que 0 assiste. 0 enfermeiro, especificamente busca nas teorias, subsídios para realizar esta tarefa da melhor maneira possível. Observa-se que as teorias selecionadas se aplicam ao processo de reabilitação em todas as suas etapas. Wanda Horta enfatiza a primeira parte deste processo: as necessi dades básicas e, tanto Callista R oy como D orothea Orem utilizam a percepção em suas estruturas conceituais, por estabel ecerem conexão entre os quatro grandes conceitos das Teorias de Enfermagem: o ser 
humano, a saúde, o meio ambiente e a enfermagem ${ }^{(27)}$. A lém disso R oy, O rem e Horta abordam o pensamento holístico (28), essencial à prática de enfermagem na reabilitação.

A ssim, fundamentadas nas teorias referenciadas, 0 enfermeiro está preparado para prestar uma assistência embasada cientificamente, servindo como base para a implantação da SAE (Sistematização da A ssistência de Enfermagem), visando uma melhor qual idade e continuidade na assistência de enfermagem ao paciente em reabilitação.

ABSTRACT: Theoretical study aiming to correlate the following nursing theories: Wanda Horta's Basic Human Needs Theory, Dorothea Orem's Self-care Theory, and A daptation Calista Roy's Adaptation Theory to the rehabilitation process of people with suffering from spinal cord injury. The definition of a theoretical landmark is a complex process but al so essential for the quality of nursing care rendered to these patients who face the need for independence recovery, social readaptation and mental heal th re-establishment. Thus, the decision to ground their nursing practice on nursing theories is very important to rehabilitation nurses.

KEY W ORDS: Rehabilitation nursing; Nursing theory; Nursing models.

RESUM EN: Este es un estudio teórico que tiene el objetivo de proponer una correlación de la Teoría de N ecesidades Humanas B ásicas (Wanda Horta), Teoría del A utocuidado (Dorothea Orem) y Teoría de A daptación (Callista Roy) con el proceso de la rehabilitación de personas con lesión medular. La definición de un hito conceptual es un proceso complejo pero esencial para la calidad de la asistencia de enfermería a esos pacientes que necesitan de recuperación de su independencia y readaptación social y mental. La decisión de basar nuestra práctica de enfermería en las teorías de enfermería es un muy importante.

PA LABRAS C L AVE : Enfermería en rehabilitación; Teoría de enfermería; M odelos de enfermería.

\section{REFERENCIAS}

1. Delisa J A et al. Tratado de medicina de reabilitação: princípios e prática. 3. ed. São Paulo: M anole; 2002.

2. Lianza S. M edicina de reabilitação. 3. ed. Rio de Janeiro: Guanabara Koogan; 2001

3. A Ifaro-L efreve R. A plicação do processo de enfermagem: um guia passo a passo. 4. ed. Porto A legre: A rtes M édicas; 2000.

4. Thomaz VA, Guidardello EB. Sistematização da assistência de enfermagem: problemas identificados pelos enfermeiros. Nursing 2002; $5(54): 28-34$

5. M ujica S, Soledad M. Teorias y modelos de enfermería. Enfermería
1985: 19(83): 13-9.

6. Souza M F. A s classificações e a construção do conhecimento na enfermagem. Rev Bras Enferm 2002; 55(6): 691-6.

7. Santos EKA . Comparação entre as teorias de enfermagem de Horta, King, Rogers, Roy e Orem. Rev Paul Enferm 1985; 5(1): 3-7.

8. Dupas G, Pinto IC, M endes M D, B enedini Z. Reflexão e síntese acerca do modelo do autocuidado de Orem. Acta Paul Enferm 1994; 7(1): $19-26$.

9. LoBiondo-Wood G, Haber J. Pesquisa em enfermagem: métodos, avaliação crítica e utilização. 4. ed. Rio de J aneiro: Guanabara K oogan; 2001.

10. Carraro TE. M arco conceitual: subsídio para a assistência de enfermagem. Cogitare Enferm 1998: 3(2): 105-8.

11. Toniolli ACS, Pagliuca L M F. A nálise da aplicabilidade da teoria de Orlando em periódicos brasileiros de enfermagem. Rev B ras Enferm 2002; 55(5): 489-94.

12. Landim FLP, Frota M A, Pagliuca LM F. Teorias de enfermagem: reflexão como instrumento de construcão da crítica ao conhecimento elaborado. Cogitare Enferm 2001; 6(1): 50-6.

13. L eitão GCM , Linard AG, Rodrigues DP. Conceitos de enfermagem segundo Roy, Orem e Watson. A cta Paul Enferm 2000; 13(3): 76-80.

14. K letemberg DF. A pedagogia problematizadora no ensino do referencial teórico de Wanda Horta [monografia especialização]. D epartamento de Enfermagem, Universidade Federal do Paraná; 2002.

15. Nóbrega M M L, Barros A LBL. M odelos assistenciais para a prática de enfermagem. Rev Bras Enferm 2001; 54(1): 74-80.

16. M azzei N . N uevos modelos conceptuales. Temas Enferm A ctual 1997; 5(24): 22.

17. Rodrigues M F. Buscando um marco conceitual e uma metodologia baseados em Imogene K ing: experiência com clientes adultos diabéticos [dissertação]. Florianópolis: Universidade Federal de Santa Catarina; 1992.

18. Trentini M , Dias LPM . Conflitos na construção do conhecimento na enfermagem: uma controvérsia persistente. Texto \& Contexto Enferm 1997; 6(3): 193-208

19. George JB. Teorias de enfermagem: os fundamentos à prática profissional. 4. ed. Porto A legre: A rtes M édicas Sul; 2000.

20. Roy SC. Introduction to nursing and adaptation model. 2. ed. Englewood Cliffs: Prentice-Hall; 1984.

21. SilvaA L, A rruda EN. Referências com base em diferentes paradigmas: problema ou solução para a prática de enfermagem? Texto \& Contexto Enferm 1993; 2(1): 82-92.

22. Leopardi MT. Teoria em enfermagem: instrumentos para a prática. Florianópolis: Papa-livros; 1999.

23. Horta WA. Processo de enfermagem. São Paulo: EPU; 1979.

24. Nunes A M P, Zagonel IPS. Cuidado humano e autocuidado: contribuição de $O$ rem e Watson ao conhecimento da enfermagem. Cogitare Enferm 1996; 1(1): 28-33. 
25. Allison SE, M CL aughlin-Renpenning KE. Nursing administration in the 21 $1^{\text {st }}$ century: a self-care theory approach. Califórnia: Sage; 1998.

26. Alligood M R, M arriner-Tomey A M. Nursing theory: utilization e application. St. Louis: M osby; 2002.

27. Silveira M FA, M antovani M F, Costa M LA S, Ciccacio SR, Silva M JP. Percepção da realidade: uma abordagem conceitual para a enfermagem. Nursing 1999; 2(15):15-21.

28. Lopes Neto $D, N$ óbrega M ML. Holismo nos modelos teóricos de enfermagem. Rev Bras Enferm 1999; 52(2): 233-42.

29. Gonzáles, RM B. Cenários de cuidado: aplicação de teorias de enfermagem. Santa M aria: Pallotti; 1999.

ENDEREC DOSAUTORES:

Rua Pereira Valente, 640 - ap. 1902

Fortaleza-CE

60160-250

janaina_vall@yahoo.com.br 